\title{
Relato da situação dos modos de ensino em cursos de medicina no estado de Goiás durante a pandemia
}

\author{
Jardel de Almeida Monteiro ${ }^{1}$ \\ Monique Costa Dias ${ }^{2}$ \\ Geovanna Ribeiro Athie ${ }^{3}$ \\ Júlia Lisboa Mendes ${ }^{4}$ \\ Juliete Teresinha Silva ${ }^{5}$
}

\section{RESUMO:}

Em março de 2020, após a confirmação dos primeiros casos da COVID-19 em Goiás, medidas foram adotadas para a contenção da disseminação dessa doença. Com isso, as aulas presenciais foram suspensas em todo o estado, o que afetou a dinâmica da educação médica nas diferentes escolas médicas do estado. Considerando a importância de compilar informações a respeito dos modos digitais e híbridos de ensino nos cursos de medicina goianos, foi realizada uma procura por informações disponíveis online pelas instituições de ensino superior sobre a situação de ensino durante a pandemia. Em geral, os cursos de medicina pagos aderiram às ferramentas digitais em substituição às aulas presenciais primeiro que os cursos gratuitos das instituições públicas. Outro aspecto considerável é que o regime híbrido instituído pela maioria das instituições a partir do segundo semestre de 2020 restringiu-se às instituições pagas e à Universidade Federal de Goiás. Diante do contexto, diversos impactos sobre o ensino superior em medicina são de suma importância serem discutidos. Entre as sugestões de como minimizar o impacto pedagógico, encontra-se o diálogo aberto e horizontal com os alunos para que as demandas e eventuais problemas de aprendizagem sejam sanados em tempo hábil.

Palavras-chave: Educação Médica. Ensino online. Isolamento Social.

\section{Report on the situation of teaching modes in medical courses in the state of Goiás during the pandemic}

\begin{abstract}
:
In March 2020, after the confirmation of the first case of covid-19 in Goiás, measures were taken to contain the spread of this disease. As a result, presential classes were suspended throughout the state, which affected the dynamics of medical education in the different medical schools in the state. Considering the importance of compiling information about digital and hybrid teaching modes in medical courses in Goiás, a search was made for information available online by undergraduate education institutions about the teaching situation during the pandemic. In general, paid medical courses adhered to digital tools instead of presential classes first than free courses from public institutions. Another considerable aspect is that the hybrid regime instituted by most institutions from the second semester of 2020 was restricted to paid institutions and the Federal University of Goiás. About this scenario, several impacts on higher education in medicine are of paramount importance to be discussed. Among the suggestions on how to minimize the pedagogical impact, there is an open and horizontal dialogue with the students so that the demands and possible learning problems are solved in a timely manner.
\end{abstract}

Keywords: Medical education. eLearning. Social Isolation.

\footnotetext{
${ }^{1}$ Acadêmico de Medicina na Universidade Federal de Jataí (UFJ). E-mail: jardelmonteiro@ discente.ufj.edu.br

2 Acadêmica de Medicina na Universidade Federal de Jataí (UFJ). E-mail: monique_diass@discente.ufj.edu.br

${ }^{3}$ Acadêmica de Medicina na Universidade Federal de Jataí (UFJ). E-mail: geovannaathie@ discente.ufj.edu.br

${ }^{4}$ Acadêmica de Medicina na Universidade Federal de Jataí (UFJ). E-mail: julialisboam @ discente.ufj.edu.br

${ }^{5}$ Docente do curso de Medicina na Universidade Federal de Jataí (UFJ), mestre em Ensino na Saúde, Ginecologista

e Obstetra: julieteteresinha@ufj.edu.br
} 


\section{INTRODUÇÃO}

Em fevereiro de 2020, foi declarada pela Organização Mundial de Saúde (OMS) a pandemia da COVID-19 após a confirmação da ocorrência e transmissão disseminada da infecção pelo novo coronavírus (SARS-CoV-2). No dia 12 de março do mesmo ano, o primeiro caso da doença foi confirmado em Goiás e no dia seguinte já foi decretada situação de emergência na saúde pública do estado. Na tentativa de conter o surto do novo coronavírus, foi preciso seguir o exemplo de medidas adotadas por outros países, tais como o isolamento social e a suspensão das atividades presenciais de escolas e universidades em instituições de ensino. Segundo dados da UNESCO (2020), cerca de 85 países paralisaram as atividades educacionais presenciais, sendo que o Brasil está entre os países com o período mais prolongado de fechamento das escolas.

Entre os afetados por essas medidas, encontram-se os 16 cursos de medicina em funcionamento no estado de Goiás, que oferecem anualmente aproximadamente 1.500 vagas em diversos municípios goianos, conforme representado na Figura 1. Destas, 220 são ofertadas pelas três universidades federais no estado; 24 são pela Universidade Estadual de Goiás; e as outras resultam da oferta de 12 instituições pagas.

Assim, as regiões com curso de medicina, atualmente, e suas respectivas instituições são:

1. Região Metropolitana de Goiânia (Goiânia, Aparecida de Goiânia e Trindade)

a. Universidade Federal de Goiás (UFG);

b. Pontifícia Universidade Católica de Goiás (PUC-GO);

c. Centro Universitário de Mineiros (UNIFIMES) - Trindade;

d. Centro Universitário Alfredo Nasser (UNIFAN) - Aparecida de Goiânia;

e. Universidade de Rio Verde (UniRV) - Aparecida de Goiânia.

2. Anápolis

a. Centro Universitário de Anápolis (UniEVANGÉLICA)

3. Rio Verde

a. Universidade de Rio Verde (UniRV)

4. Jataí

a. Universidade Federal de Jataí (UFJ)

5. Mineiros

a. Centro Universitário de Mineiros (UNIFIMES)

b. Faculdade Morgana Potrich (FAMP) 
6. Itumbiara

a. Universidade Estadual de Goiás (UEG)

b. Centro Universitário IMEPAC Itumbiara

7. Catalão

a. Universidade Federal de Catalão (UFCAT)

8. Goiatuba

a. Centro Universitário de Goiatuba (UniCerrado)

9. Goianésia

a. Universidade de Rio Verde (UniRV) - Goianésia

10. Formosa

a. Universidade de Rio Verde (UniRV) - Formosa

Para entender como o estado de Goiás conseguiu instituir essas escolas, precisamos olhar para a história da medicina em Goiás. Em 1952, a então recém-criada Associação Médica de Goiás (AMG) realizou um estudo o qual mostrou que dos 179 municípios goianos, apenas 21 tinham médicos. No mesmo ano, o médico Francisco Ludovico de Almeida Neto foi responsável pelas primeiras ações na criação de uma faculdade de medicina em Goiás. Para acelerar o processo, a AMG criou em 1957 a Associação Pró-Faculdade de Medicina de Goiás, buscando a implementação de novas escolas médicas no estado para suprir a alta demanda por profissionais. No ano seguinte, o governo fez uma doação do terreno no Setor Universitário onde hoje está o Hospital das Clínicas da Universidade Federal de Goiás. No ano de 1960, foi autorizado pelo governo federal o funcionamento da Faculdade e o primeiro vestibular aconteceu no mesmo ano, com 179 inscritos. Segundo pesquisa Demografia Médica realizada pela Faculdade de Medicina da Universidade de São Paulo (USP), Goiás já contava com 13.360 médicos em 2018, uma proporção de 1,97 profissionais por mil habitantes, 10\% a menos do que a média nacional, ressaltando ainda mais a importância da formação e a especialização de novos profissionais no estado (SCHEFFER, 2018).

Considerando esse cenário, as escolas médicas goianas não deixariam de ser afetadas pela decisão do Ministério da Educação que autorizou a inserção da Medicina dentre os cursos que poderiam utilizar o Ensino Remoto, por meio da portaria n ${ }^{\circ} 345$ do dia 19 de março de 2020 (BRASIL, 2020), que autorizou em caráter excepcional, especificamente para o curso de Medicina, a substituição das disciplinas presenciais teóricas-cognitivas do primeiro ao quarto ano do curso por meio de tecnologias da informação e comunicação. Sendo assim, como as demais faculdades do país, o ensino médico goiano teve que se adaptar à distância e à necessidade de utilização das plataformas e ferramentas digitais, em sua maioria de maneira 
rápida. A paralisação das atividades desses cursos e principalmente das práticas que são intrínsecas à formação médica gerou sérios impactos não só no ensino desses estudantes como também nas próprias comunidades que se beneficiam das atividades desenvolvidas por esses alunos.

Considera-se oportuno e de extrema importância relatar um tema pouco abordado, até então, com escassos achados bibliográficos disponíveis sobre a experiência de ensino em cursos de medicina no estado de Goiás durante a pandemia (BARBOSA et al., 2020). Sendo assim, é preciso um agrupamento de informações para entender as particularidades do estado de Goiás e diminuir os impactos do ensino online na formação médica.

O presente trabalho consiste em uma revisão narrativa documental da situação do ensino médico do estado de Goiás durante a pandemia causada pela COVID-19, realizada com o intuito de identificar a experiência de ensino durante a pandemia do novo coronavírus por cada instituição médica do estado. Diante disso, foi feita uma pesquisa dos documentos disponibilizados nos anos de 2020 e 2021 nas plataformas digitais e sítios oficiais das instituições que oferecem o curso de medicina em abril de 2021, com o objetivo de buscar informações sobre cada unidade e identificar a situação educacional a qual se encontra cada unidade.

\section{O ENSINO MÉDICO DURANTE A PANDEMIA EM GOIÁS}

O distanciamento social imposto pela pandemia da COVID-19 implicou em alterações na dinâmica como os cursos de medicina funcionam no Estado de Goiás. Diante das circunstâncias sanitárias, a Secretaria de Estado da Saúde de Goiás (2020) determinou a suspensão de todas as atividades de ensino presenciais de todas as instituições educacionais do estado em 15 de março de 2020. Tal medida implicou em profundas mudanças no processo de ensino-aprendizagem não só dos cursos superiores da área da saúde, mas de todo o sistema de ensino.

Em um primeiro momento, as instituições organizaram-se para a execução do Ensino Remoto Emergencial (ERE) para os cursos. Posteriormente, foi necessária uma articulação para que as atividades práticas laboratoriais e em serviços de saúde retomassem, incluindo o estágio curricular obrigatório, o internato.

As instituições de ensino superior reagiram de forma e em tempos diferentes para a adoção do ensino remoto e a utilização de recursos digitais para as atividades de ensino. Em um primeiro momento, as universidades federais do estado não aderiram ao ensino remoto. A 
Universidade Federal de Goiás (2020), após suspender as aulas em março de 2020, retomou as atividades de ensino de graduação no final de agosto do mesmo ano por meio do Ensino Remoto Emergencial (ERE), usando ferramentas digitais. Quanto às atividades práticas presenciais, após a publicação do Protocolo de biossegurança para retorno das atividades nas Instituições Federais de Ensino pelo MEC (2020) e o Protocolo de Biossegurança para Retorno das Atividades Presenciais nas Instituições de Ensino do Estado de Goiás da Secretaria de Estado de Saúde de Goiás (2020), foi aprovado o retorno das Atividades Práticas Emergenciais (APEs) para o semestre letivo de 2020/1 para serem realizadas a partir de dezembro (UNIVERSIDADE FEDERAL DE GOIÁS, 2020), adotando um regime híbrido de ensino, em que conteúdos e disciplinas teóricas eram ministrados on-line e as atividades práticas em laboratórios e serviços de saúde aconteciam conforme protocolo de biossegurança da instituição. As recém-criadas (supernovas) Universidade Federal de Jataí (UFJ) e Universidade Federal de Catalão (UFCAT) também reagiram de forma semelhante quanto ao Ensino Remoto no segundo semestre de 2020, mas não quanto às APEs.

A UFJ, após a suspensão das aulas na metade de março, suspendeu o calendário acadêmico de todos os cursos de graduação em 27 de março de 2020, publicizando a não adesão ao ensino remoto emergencial pela instituição naquele momento (UNIVERSIDADE FEDERAL DE JATAÍ, 2020). Em outubro de 2020, o curso de medicina retomou as atividades de ensino exclusivamente por ferramentas digitais. Até abril de 2021, as atividades dos quatro primeiros anos do curso permaneceram exclusivamente pelos meios tecnológicos do ERE. Não foi regulamentada nenhuma atividade prática presencial, nenhum protocolo de biossegurança público ou qualquer planejamento de retorno prático em 2020 e tampouco até abril de 2021, com exceção das atividades do Internato (UNIVERSIDADE FEDERAL DE JATAÍ, 2020).

A UFCAT, por sua vez, aderiu ao ERE em período semelhante à UFG. Meses após suspender o calendário acadêmico dos cursos de graduação em 27 de março de 2020 (UNIVERSIDADE FEDERAL DE CATALÃO, 2020), aprovou a retomada das atividades de ensino de forma exclusivamente remotas a partir de agosto de 2020 em um período suplementar especial (UNIVERSIDADE FEDERAL DE CATALÃO, 2020). Embora não tenha realizado atividades práticas presenciais, em dezembro de 2020, foi publicado o protocolo de biossegurança e as recomendações para um eventual retorno para atividades práticas (UNIVERSIDADE FEDERAL DE CATALÃO, 2020).

Ainda na esfera das universidades públicas gratuitas do estado, o curso de medicina da Universidade Estadual de Goiás (2020) seguiu as normativas e instruções da instituição. De forma que, antes que as instituições federais, retomou atividades de ensino em modalidade 
exclusivamente remota ainda no primeiro semestre de 2020. Até março de 2021, ofertava atividades on-line, enquanto um plano para um eventual regime híbrido de atividades estava em construção.

Os cursos de medicina ofertados pela Universidade de Rio Verde (UniRV) desenvolveram as atividades de ensino de forma semelhante. A UniRV oferta o curso de medicina em quatro municípios goianos: Rio Verde, Aparecida de Goiânia, Formosa e Goianésia. Já no início de abril de 2020, regulamentou e implementou o uso de ferramentas digitais em substituição às aulas presenciais (UNIVERSIDADE DE RIO VERDE, 2020). O estágio obrigatório do internato retomou no mês de julho de 2020 (UNIVERSIDADE DE RIO VERDE, 2020). Já as atividades práticas presenciais dos outros períodos do curso foram regulamentadas para serem realizadas a partir de setembro de 2020 (UNIVERSIDADE DE RIO VERDE, 2020). Em março de 2021, suspendeu as atividades práticas presenciais, regulamentando o seu retorno para o dia 19 de abril de 2021 (UNIVERSIDADE DE RIO VERDE, 2021).

Assim como a UniRV, o Centro Universitário de Mineiros, a UNIFIMES é uma instituição de ensino superior pública não gratuita. Assim, ela oferta cursos de medicina em dois municípios goianos: Mineiros e Trindade. Diante da pandemia, as unidades da UNIFIMES reagiram de forma semelhante. Foram instituídas férias com a suspensão das aulas em março de 2020. Retomaram as atividades por ensino remoto ainda no início de abril de 2020 (CENTRO UNIVERSITÁRIO DE MINEIROS, 2020). Em 2021, retomaram as atividades práticas presenciais em fevereiro (CENTRO UNIVERSITÁRIO DE MINEIROS, 2021), suspenderam-nas por conta da situação epidemiológica em março e prorrogaram a suspensão para o início de abril de 2021 (CENTRO UNIVERSITÁRIO DE MINEIROS, 2021).

Semelhantemente à UniRV e à UNIFIMES, os cursos de instituições privadas do estado de Goiás adotaram rapidamente o uso de ferramentas digitais e o ERE. Todos iniciaram suas atividades remotas no primeiro semestre de 2020 e, em sua grande maioria, em menos de um mês após a suspensão das aulas na metade de março.

A Pontifícia Universidade Católica de Goiás (PUC-GO), por exemplo, já no dia 16 de março de 2020, comunicou o uso de ambientes virtuais de aprendizado por conta das aulas presenciais suspensas, comunicando um reagendamento das atividades práticas para realização futura (PONTIFÍCIA UNIVERSIDADE CATÓLICA DE GOIÁS, 2020). As atividades do internato retornaram em junho de 2020 com os protocolos sanitários de segurança, conforme o Sindicato das Entidades Mantenedoras de Estabelecimentos de Educação Superior do Estado de Goiás (2020). Já os quatro primeiros anos do curso retomaram as atividades de forma híbrida 
desde o segundo semestre de 2020 com rodízio de alunos e redução de turmas para aulas práticas (PUC TV GOIÁS, 2020). Após alteração da situação epidemiológica, suspendeu atividades práticas presenciais, substituindo-as por atividades remotas em março de 2021, para retorno presencial em abril de 2021 (PONTIFÍCIA UNIVERSIDADE CATÓLICA DE GOIÁS, 2021).

Situado em Aparecida de Goiânia, o Centro Universitário Alfredo Nasser (UNIFAN) instituiu, no dia 31 de março de 2020, o regime remoto de ensino, conforme determinações. No segundo semestre de 2020, retomou as atividades práticas presenciais, adotando um regime híbrido de ensino (CENTRO UNIVERSITÁRIO ALFREDO NASSER, 2020). Em março de 2021, suspenderam as atividades práticas por conta dos decretos municipais, e o retorno foi aprovado para que atividades práticas retomassem dia 19 de abril de 2021 (CENTRO UNIVERSITÁRIO ALFREDO NASSER, 2021).

O Centro Universitário de Anápolis (UniEVANGÉLICA) retomou as atividades de graduação já no dia 19 de março de 2020 por ERE (ASSOCIAÇÃO EDUCATIVA EVANGÉLICA, 2020). Aprovou em 19 de junho de 2020 a retomada das atividades do Internato de Medicina, com uso de EPIs e manteve as demais atividades práticas substituídas por recursos digitais (ASSOCIAÇÃO EDUCATIVA EVANGÉLICA, 2020). Estabeleceu atividades práticas essenciais presenciais no segundo semestre de 2020. Além das práticas, iniciou o primeiro semestre letivo de $2021 \mathrm{com}$ aulas presenciais, que foram suspensas no dia 15 março (ASSOCIAÇÃO EDUCATIVA EVANGÉLICA, 2021). No dia 10 de abril, aprovou o retorno das atividades práticas presenciais para o dia 13 de abril (ASSOCIAÇÃO EDUCATIVA EVANGÉLICA, 2021), já dia 14 de abril comunicou o retorno imediato das aulas presencialmente com limite de $30 \%$ de lotação por ambiente (ASSOCIAÇÃO EDUCATIVA EVANGÉLICA, 2021).

Em Mineiros, a Faculdade Morgana Potrich (FAMP) instituiu ensino remoto ainda no primeiro semestre. Adotou regime híbrido com aulas práticas presenciais a partir de setembro de 2020 (CENTRO DE ENSINO SUPERIOR MORGANA POTRICH EIRELI, 2020). Em março de 2021, suspendeu as atividades práticas presenciais e prorrogou a suspensão para o mês de abril (CENTRO DE ENSINO SUPERIOR MORGANA POTRICH EIRELI, 2021).

O Centro Universitário de Goiatuba (UniCerrado) retomou as atividades de forma híbrida com as teóricas remotamente e práticas presenciais em agosto de 2020; suspendeu as atividades práticas presenciais em março de 2021 para retorno em abril de 2021 (CENTRO UNIVERSITÁRIO DE GOIATUBA, 2021). 
A Faculdade IMEPAC de Itumbiara, imediatamente após a suspensão das aulas presenciais em março de 2020, implementou o uso de ambientes de aprendizado virtuais para execução do ensino remoto e só retomadas no segundo semestre de 2020 (CENTRO UNIVERSITÁRIO IMEPAC, 2020).

Nessas circunstâncias, o ensino médico durante a pandemia é um grande desafio, haja vista que exige que os acadêmicos se adaptem às novas tecnologias e às novas metodologias de ensino, as quais aproveitam as ferramentas digitais para compensar os prejuízos no ensino.

De acordo com o estudo transversal realizado com 656 universitários do curso de medicina de diversas universidades de todas as regiões do Brasil, o ensino remoto adaptado ao cenário de isolamento social no enfrentamento da pandemia de SARS-COV-2 trouxe grande prejuízo no aprendizado. Além disso, foi apresentado que 79,4\% dos estudantes apontaram baixa adaptação ao ensino à distância (EAD) e, destes, 78,5\% acreditavam que não tinham um aprendizado adequado (TEXEIRA et al., 2021).

Ainda sobre o estudo, foi apontado alguns fatores que favoreceram o prejuízo acadêmico dos alunos. O principal contribuinte analisado foi o sofrimento psíquico que os discentes enfrentavam (62,8\%). Foram listados os principais sintomas somáticos decorrentes: sono comprometido $(54,6 \%)$, desconforto estomacal $(51,7 \%)$ e cefaleia $(49,2 \%)$. Além disso, relataram má digestão $(36,4 \%)$, falta de apetite $(27 \%)$ e tremores nas mãos $(23,3 \%)$. Assim, foi possível concluir que esses fatores citados, somados aos fatores ambientais a que os entrevistados estavam submetidos, resultaram no comprometimento do ensino, resultando em: dificuldade de concentração em aulas remotas $(66,8 \%)$ e ausência de aprendizado com EAD $(39,2 \%)$. Por fim, o artigo demonstrou que o sofrimento mental se apresenta ainda mais elevado na comunidade discente do curso de medicina durante a pandemia da COVID-19, o que resulta em grande prejuízo acadêmico (TEXEIRA et al., 2021).

Segundo os dados disponibilizados pelo Instituto Brasileiro de Geografia e Estatística (IBGE), por meio da Pesquisa Nacional por Amostra de Domicílios Contínua, apenas 79,1\% dos domicílios brasileiros têm acesso à internet no ano de 2018. Porém, essa taxa de acesso na comunidade discente do curso de medicina é consideravelmente maior mesmo nas universidades públicas, como foi apontado no estudo transversal descritivo realizado na Universidade Federal do Pará, em que 93,5\% dos alunos entrevistados relataram ter acesso à internet (REGO et al, 2018). Entretanto, apesar de apresentarem acesso a esse recurso tecnológico, a pesquisa realizada na Universidade Federal do Delta do Parnaíba no Piauí apontou que menos da metade dos discentes com acesso à internet tem uma velocidade de navegação minimamente adequada para o ensino remoto. Somado a isso, aqueles indivíduos 
que residem em zonas rurais ou interioranas tinham menor acesso à internet $\mathrm{e}$, ainda mais, a velocidade adequada de navegação. Isso dificulta consideravelmente o aprendizado, na medida que nas transmissões ao vivo são necessárias altas taxas de transferência de dados para a obtenção de imagem e áudio de qualidade (SILVA, et al., 2021).

Ainda sobre o estudo transversal realizado em todo o âmbito nacional, é possível identificar a aflição e a ansiedade dos indivíduos participantes em relação ao medo de contrair o vírus $(59,3 \%)$, somado à preocupação da convivência com indivíduos que trabalham fora de casa $(53,7 \%)$. Ademais, a apreensão dos alunos que residem com a família e/ou o parceiro afetivo e necessitam realizar práticas acadêmicas em ambientes expostos à contaminação ao novo coronavírus. Diante disso, esses fatores contribuem para o adoecimento mental e o prejuízo do ensino, na medida que a angústia está presente no ambiente residencial e de prática acadêmica (TEXEIRA et al., 2021).

Porém, em interface do prejuízo acadêmico e adoecimento mental, o cenário atual de pandemia e de ensino remoto trouxe muitas oportunidades de mudanças, aprimoramento e desenvolvimento de metodologias de ensino. No futuro, a educação à distância pode ser consolidada e tornar-se complementar ao método de ensino atual, tornando-se parte do currículo institucional. Somado a isso, um mecanismo tecnológico flexível e acessível facilita a aceitação dos discentes pertencentes a uma geração que se desenvolveu no mesmo período das Tecnologias da Informação e Comunicação (TIC). Esse cenário pode refletir no futuro da telemedicina brasileira, levando atendimento médico generalista e especializado para diversas regiões nacionais, o que promoverá o aumento significativo do acesso à saúde (SILVA et al., 2021).

\section{CONSIDERAÇÕES FINAIS}

É perceptível que as instituições públicas gratuitas tiveram atuações diferentes das instituições pagas. Exemplo claro disso é que as instituições federais adotaram o regime de ensino remoto apenas no segundo semestre de 2020. Além disso, em relação ao regime híbrido e às atividades práticas presenciais, com exceção da UFG, apenas as instituições de ensino pagas do estado tiveram regime de ensino híbrido desde o início da pandemia. A UFCAT e a UEG possuem planos de retorno presencial, a depender da situação epidemiológica de seus respectivos municípios. É provável que a possibilidade de atividades práticas presenciais associadas ao ERE seja cada vez mais cogitada por essas instituições públicas no decorrer de 2021. Até o início de abril de 2021, a única instituição sem documento e/ou informações 
públicas sobre o retorno presencial para atividades práticas essenciais não adaptáveis ao ensino remoto era a UFJ.

Entre as limitações do presente trabalho, pode-se destacar possíveis desencontros entre as informações e documentações publicadas pelas instituições e a execução de tais. Além disso, não foi objetivo deste trabalho avaliar as atividades pedagógicas desenvolvidas pelas instituições e nem a qualidade dessas. Dessa maneira, são necessárias pesquisas que avaliem as percepções e a qualidade do ensino médico durante a pandemia.

Por fim, a circunstância atual resultou em diversas incertezas sobre o cenário de aprendizado teórico, prático e de perspectiva para a formatura dos estudantes de medicina goianos. Diante disso, o contexto do ensino médico no estado de Goiás encontra-se com impacto pedagógico significativo por questões de diferenças estruturais e socioambientais, como foi aqui apresentado. Portanto, sugere-se a execução de eventos informativos em parceria com as representações estudantis como uma maneira de sugestionar alternativas educativas, a fim de popularizar meios de utilização do tempo livre para autocuidado e enriquecimento do currículo e formação crítica, reflexiva e humanitária dos alunos. Somado a isso, o protagonismo estudantil nas decisões institucionais é de suma importância para que as adequações dos mecanismos de ensino sejam feitas e as demandas acadêmicas específicas dos cursos sejam sanadas de maneira mais efetiva, garantido melhor aproveitamento educacional e formação médica no atual contexto de pandemia. 


\section{REFERÊNCIAS BIBLIOGRÁFICAS}

ASSOCIAÇÃO EDUCATIVA EVANGÉLICA. Comunicado Importante de 14 de abril de 2021. Anápolis, 14 abr. 2021. Disponível em: <https://www4.unievangelica.edu.br/noticia/comunicado-covid>. Acesso em: 18 abr. 2021.

ASSOCIAÇÃO EDUCATIVA EVANGÉLICA. Gabinete de Gestão de Crise - Centro Universitário de Anápolis. Comunicado de 16 de março de 2020. Anápolis, 16 mar. 2020. Disponível em: <https://www4.unievangelica.edu.br/storage/3375/Comunicado03_2020.pdf>. Acesso em: 18 abr. 2021.

ASSOCIAÇÃO EDUCATIVA EVANGÉLICA. Gabinete de Gestão de Crise - Centro Universitário de Anápolis. COMUNICADO OFICIAL - 19/06/2020. Anápolis, 19 jun. 2020. Disponível em: <https://www4.unievangelica.edu.br/noticia/comunicado-oficial-retorno-dasaulas-praticas-presenciais>. Acesso em: 18 abr. 2021.

ASSOCIAÇÃO EDUCATIVA EVANGÉLICA. Gabinete de Gestão de Crise - Centro Universitário de Anápolis. Comunicado de 15 de março de 2021. Anápolis, 15 mar. 2021. Disponível em: <https://www4.unievangelica.edu.br/storage/4668/TIMBRADO-AEE---74Anos.pdf>. Acesso em: 18 abr. 2021.

ASSOCIAÇÃO EDUCATIVA EVANGÉLICA. Gabinete de Gestão de Crise - Centro Universitário de Anápolis. Comunicado de 10 de abril de 2021. Anápolis, 10 abr. 2021. Disponível em: <https://www4.unievangelica.edu.br/storage/4779/comunicado__EST\%C3\%81GIOS_E_PR\%C3\%81TICAS_LABORATORIAIS.pdf >. Acesso em: 18 abr. 2021.

BARBOSA, Ana Flávia Cândido et al. Como a COVID-19 mudou a forma de educação: um relato de experiência. RESU - Revista Educação em Saúde: ANAIS DA 18ª MOSTRA DE SAÚDE, Anápolis, v. 8, ed. Suplemento 1, 22 jun. 2020. Disponível em: http://periodicos.unievangelica.edu.br/index.php/educacaoemsaude/article/view/4598. Acesso em: 10 abr. 2021. 
BRASIL. Ministério da Educação (Brasília). Portaria n ${ }^{\circ}$ 345. [S. l.], 19 mar. 2020. Disponível em: <https://www.in.gov.br/en/web/dou/-/portaria-n-345-de-19-de-marco-de-2020248881422 ?inheritRedirect $=$ true $\&$ redirect $=\% 2 \mathrm{Fweb} \% 2 \mathrm{Fguest} \% 2 \mathrm{Fsearch} \% 3 \mathrm{FqSearch} \% 3 \mathrm{DPo}$ rtaria\%2520345\%2520de\%252019\%2520de\%2520mar\%25C3\%25A7o\%2520de\% 25202020 >. Acesso em: 18 abr. 2021.

CENTRO DE ENSINO SUPERIOR MORGANA POTRICH EIRELI. Direção Geral da Faculdade Morgana Potrich. COMUNICADO INSTITUCIONAL. Mineiros, 14 set. 2020. Disponível em: <https://fampfaculdade.com.br/famp/institucional/copem/>. Acesso em: 18 abr. 2021.

CENTRO DE ENSINO SUPERIOR MORGANA POTRICH EIRELI. Direção Geral da Faculdade Morgana Potrich. COMUNICADO INSTITUCIONAL de 12 de abril de 2021. Mineiros, 12 abr. 2021. Disponível em: <https://fampfaculdade.com.br/wpcontent/uploads/2021/04/Prorrogacao-das-ferias-12-04-21-portaria.pdf/>. Acesso em: 18 abr. 2021.

CENTRO UNIVERSITÁRIO ALFREDO NASSER. Notícias UNIFAN. Direção da Unifan se reúne com coordenadores de cursos para apresentar resolução com novas normas de funcionamento. Aparecida de Goiânia, 2020. Disponível em: <https://www.unifan.edu.br/unifan/aparecida/?p=2434>. Acesso em: 18 abr. 2021.

CENTRO UNIVERSITÁRIO ALFREDO NASSER. Notícias UNIFAN. Faculdade Alfredo Nasser retoma atividades práticas laboratoriais e ambulatoriais dos cursos de Saúde. Aparecida de Goiânia, entre 2020 e 2021. Disponível em: <https://www.unifan.edu.br/unifan/aparecida/?p=2687>. Acesso em: 18 abr. 2021.

CENTRO UNIVERSITÁRIO DE GOIATUBA . Reitoria do Centro Universitário de Goiatuba. Portaria $\mathbf{n}^{\circ}$ 015, de 16 de março de 2021. Goiatuba, 15 mar. 2021. Disponível em: <http://www.unicerrado.edu.br/site/2021/03/18/portaria-reitoria-no-015-21-de-16-de-marcode-2021/>. Acesso em: 18 abr. 2021.

CENTRO UNIVERSITÁRIO DE MINEIROS. Conselho Universitário do Centro Universitário de Mineiros - CONSUN. RESOLUÇÃO Nº 82/CONSUN/2020. Mineiros, 2 abr. 2020. 
Disponível em: <https://unifimes.edu.br/institucional/\#tab-30ddec01741ebc4c405>. Acesso em: 18 abr. 2021.

CENTRO UNIVERSITÁRIO DE MINEIROS. Reitoria do Centro Universitário de Mineiros. Portaria $\mathbf{n}^{\circ}$ 01, de 22 de janeiro de 2021. Mineiros, 22 jan. 2021. Disponível em: <https://unifimes.edu.br/institucional/\#tab-bb9c8f1547027a57ed5>. Acesso em: 18 abr. 2021.

CENTRO UNIVERSITÁRIO DE MINEIROS. Pró-Reitoria de Ensino, de Pesquisa e de Extensão. Prorrogação de Suspensão das Aulas Presenciais. [S. l.], 12 abr. 2021. Disponível em: <https://unifimes.edu.br/2021/04/12/prorrogacao-de-suspensao-das-aulas-presenciais/>. Acesso em: 18 abr. 2021.

CENTRO UNIVERSITÁRIO IMEPAC. Faculdade IMEPAC Itumbiara. Portaria Conjunta $\mathbf{n}^{\circ}$ 01/2020. Araguari/MG e Itumbiara/GO, 14 mar. 2020. Disponível em: <https://imepac.edu.br/portaria-conjunta-no-01-2020/>. Acesso em: 18 abr. 2021.

INSTITUTO BRASILEIRO DE GEOGRAFIA E ESTATÍSTICA. Pesquisa Nacional por Amostra de Domicílios Contínua. Acesso à internet e à televisão e posse de telefone móvel celular para uso pessoal - 2018. Rio de Janeiro: IBGE; 2020. Disponível em: $<$ https://biblioteca.ibge.gov.br/index.php/biblioteca-catalogo?view=detalhes\&id=2101705>. Acessado em 17 abr. 2021.

MINISTÉRIO DA EDUCAÇÃO. Secretária de Educação Superior. Protocolo de biossegurança para retorno das atividades nas Instituições Federais de Ensino. Brasília, 1 jul. 2020. Disponível em: <https://www.gov.br/mec/pt-br/centrais-de-conteudo/campanhas1/coronavirus/CARTILHAPROTOCOLODEBIOSSEGURANAR101.pdf >. Acesso em: 18 abr. 2021.

PONTIFÍCIA UNIVERSIDADE CATÓLICA DE GOIÁS. Gabinete do Reitor da Pontifícia Universidade Católica de Goiás. Comunicado - 28 de fevereiro de 2021. ORIENTAÇÕES PARA AS ATIVIDADES ACADÊMICAS NA PUC GOIÁS NO PERÍODO DE $1^{\circ}$ A 7 DE MARÇO DE 2021, Goiânia, 28 fev. 2021. Disponível em: <https://www.pucgoias.edu.br/wpcontent/uploads/2021/02/comunicado-28-02-2021.pdf>. Acesso em: 18 abr. 2021. 
PONTIFÍCIA UNIVERSIDADE CATÓLICA DE GOIÁS. Gabinete do Reitor da Pontifícia Universidade Católica de Goiás. Comunicado - 15 de março de 2020. COMUNICADO À COMUNIDADE ACADÊMICA DA PUC GOIÁS, Goiânia, 15 abr. 2021. Disponível em: $<$ https://noticias.pucgoias.edu.br/wp-content/uploads/2020/03/Comunicado-15mar20202.pdf>. Acesso em: 18 abr. 2021.

PUC TV GOIÁS. Alunos do Curso de Medicina da PUC Goiás voltam às aulas presenciais em laboratório. Intérprete: Eliara Salla. Gravação de Paulo Sérgio Machado. Goiânia: PUC TV, 2020. Disponível em: 〈https://www.youtube.com/watch?v=4a5fMJvr14Q〉. Acesso em: 18 abr. 2021.

REGO, Rhyan Meninea, et al. O perfil atual do estudante de Medicina e sua repercussão na vivência do curso. Pará Research Medical Journal, 2018. Disponível em: <https://www.prmjournal.org/article/10.4322/prmj.2018.005/pdf/prmjournal-2-1-4-e05.pdf $>$. Acessado em 17 abr. 2021.

SCHEFFER, Mário. et al. Demografia Médica no Brasil 2018. São Paulo, SP: FMUSP, CFM, Cremesp, 2018. 286 p. ISBN: 978-85-87077-55-4

SECRETARIA DE ESTADO DE SAÚDE DE GOIÁS (Goiás). Nota Técnica no : 1/2020 GAB-03076. $[S . \quad l$ l. 15 mar. 2020. Disponível em: <saude.go.gov.br/files/banner_coronavirus/notatecnica.pdf>. Acesso em: 18 abr. 2021.

SECRETARIA DE ESTADO DE SAÚDE DE GOIÁS. Protocolo de Biossegurança para Retorno das Atividades Presenciais nas Instituições de Ensino do Estado de Goiás. Goiânia, Julho $2020 . \quad$ Disponível em: <https://www.saude.go.gov.br/files/banner_coronavirus/Protocolos/Protocolo\%20de\%20retor no\%20as\%20atividades\%20presenciais\%20nas\%20institui\%C3\%A7\%C3\%B5es\%20de\%20e nsino\%20de\%20Goi\%C3\%A1s.pdf>. Acesso em: 18 abr. 2021.

SILVA, Francisco Theogenes, et al. Adaptações e repercussões nas experiências em uma universidade de educação híbrida durante a pandemia Sars-CoV-2. Scielo: 2021. Disponível em: <https://www.scielo.br/scielo.php?script=sci_arttext\&pid=S010055022021000200401\&lang=pt>. Acessado em 17 abr. 2021. 
SILVA, Pedro Henrique dos Santos, et al. Educação remota na continuidade da formação médica em tempos de pandemia: viabilidade e percepções. Scielo: 2021. Disponível em: $<$ https://www.scielo.br/scielo.php?script=sci_arttext\&pid=S010055022021000100222\&lang=pt\#B25>. Acessado em 17 abr. 2021.

SINDICATO DAS ENTIDADES MANTENEDORAS DE ESTABELECIMENTOS DE EDUCAÇÃO SUPERIOR DO ESTADO DE GOIÁS. Notícias SEMESG. Faculdades particulares retomam aulas práticas em Goiás para alunos dos últimos períodos: Pelo menos dez universidades devem reiniciar atividades. [S. l.], 29 maio 2020. Disponível em: $<$ http://www.semesg.org.br/eficiente/sites/semesg.org.br/ptbr/site.php?secao=noticias\&pub=1579> . Acesso em: 18 abr. 2021 .

TEXEIRA, Larissa de Araújo Correia, et al. Saúde mental dos estudantes de Medicina do Brasil durante a pandemia da coronavirus disease 2019. Scielo, 2020. Disponível em: $<$ https://www.scielo.br/scielo.php?script=sci_arttext\&pid=S004720852021000100021\&lang=pt>. Acessado em: 16 abr. 2021.

UNESCO. “COVID-19 Educational Disruption and Response". UNESCO Website 06 mai. 2020. Disponível em: <https://en.unesco.org/covid19/educationresponse>. Acesso em 16 abr. 2021.

UNIVERSIDADE DE RIO VERDE. Portaria n 1.303. Rio Verde, 23 jun. 2020. Disponível em: <https://www.unirv.edu.br/conteudos/fckfiles/files/Portaria\%20N_\%201_303\%20$\% 20$ Prorroga\%20o\%20regime\%20de\%20aulas\%20n\%C3\%A3o\%20presenciais.pdf $>$. Acesso em: 18 abr. 2021.

UNIVERSIDADE DE RIO VERDE. Portaria n $^{\circ}$ 1.350. Rio Verde, 29 jun. 2020. Disponível em:

<https://www.unirv.edu.br/conteudos/fckfiles/files/Portaria\%20n_\%201_350\%20Altera\%20a $\% 20$ portaria\%20n_\%201_316\%20sobre\%20retorno\%20das\%20atividades\%20de\%20Internat o.pdf>. Acesso em: 18 abr. 2021. 
UNIVERSIDADE DE RIO VERDE. Portaria $\mathbf{n}^{\circ}$ 1.881. Rio Verde, 08 set. 2020. Disponível em: <https://www.unirv.edu.br/conteudos/fckfiles/files/Portaria\%20n_\%201_8812020(1).pdf>. Acesso em: 18 abr. 2021.

UNIVERSIDADE DE RIO VERDE. Portaria $\mathbf{n}^{\circ}$ 645. Rio Verde, 14 abr. 2021. Disponível em: $\quad$ https://www.unirv.edu.br/conteudos/fckfiles/files/Portaria\%20n_\%206452021\%20Funcionamento\%20das\%20a\%20(1).pdf>. Acesso em: 18 abr. 2021.

UNIVERSIDADE ESTADUAL DE GOIÁS. Memorando nº 269/2020 - GAGC-16136. Calendário Acadêmico de 2020, Anápolis, 13 out. 2020. Disponível em: http://cdn.ueg.edu.br/source/campus_itumbiara_98/conteudo/11199/Calendario_academico_s egundo_semestre_de_2020.pdf. Acesso em: 18 abr. 2021.

UNIVERSIDADE FEDERAL DE CATALÃO. RESOLUÇÃO UFCAT N. ${ }^{*}$ 006/2020. Catalão, 27 mar. 2020. Disponível em: <https://files.cercomp.ufg.br/weby/up/519/o/Resolu\%C3\%A7\%C3\%A3o_006_de_27_de_ma r\%C3\%A7o_de_2020.pdf>. Acesso em: 18 abr. 2021.

UNIVERSIDADE FEDERAL DE CATALÃO. RESOLUÇÃO UFCAT No $\mathbf{N}^{\mathbf{0 1 0}}$ (2020. Catalão, $10 \quad$ ago. 2020.2 Disponível <https://files.cercomp.ufg.br/weby/up/519/o/Resolucao_010.pdf>. Acesso em: 18 abr. 2021.

UNIVERSIDADE FEDERAL DE CATALÃO. PROTOCOLO DE BIOSSEGURANÇA, ESPAÇO FÍSICO, PROTOCOLOS SANITÁRIOS E MONITORAMENTO DA COVID19 NA UFCAT. Catalão, 17 dez. 2020. Disponível em: <https://files.cercomp.ufg.br/weby/up/519/o/Manual_de_Bioseguran\%C3\%A7a-UFCAT__Vers\%C3\%A3o_21-12.pdf?1608647350>. Acesso em: 18 abr. 2021.

UNIVERSIDADE FEDERAL DE GOIÁS. CONSELHO UNIVERSITÁRIO DA UNIVERSIDADE FEDERAL DE GOIÁS. RESOLUÇÃO CONSUNI No 34/2020. [S. l.], 14 ago. 2020 .

Disponível em: $<$ https://sistemas.ufg.br/consultas_publicas/resolucoes/arquivos/Resolucao_CONSUNI_2020 _0034.pdf>. Acesso em: 18 abr. 2021. 
UNIVERSIDADE FEDERAL DE GOIÁS. Pró-Reitoria de Graduação da UFG. RECOMENDAÇÕES GERAIS PARA RETORNO DE ATIVIDADES PRÁTICAS EMERGENCIAIS (APEs) DA EDUCAÇÃO BÁSICA E DA GRADUAÇÃO NA UFG. Goiânia, 18 nov. 2020. Disponível em: <https://files.cercomp.ufg.br/weby/up/90/o/Recomenda\%C3\%A7\%C3\%B5es_de_biossegura n\%C3\%A7a_APES_-_UFG.pdf>. Acesso em: 18 abr. 2021.

UNIVERSIDADE FEDERAL DE JATAÍ. Conselho Universitário da Universidade Federal de Jataí. RESOLUÇÃO - CONSUNI No 001R/2020. [S. l.], 27 mar. 2020. Disponível em: <https://files.cercomp.ufg.br/weby/up/881/o/Resolu\%C3\%A7\%C3\%A3o.001.R.CONSUNI.U FJ.pdf?1617159213>. Acesso em: 18 abr. 2021.

UNIVERSITÁRIO FEDERAL DE JATAÍ. Conselho Universitário da Universidade Federal de Jataí. RESOLUÇÃO - CONSUNI No 001/2021. [S. l.], 24 mar. 2021. Disponível em: <https://files.cercomp.ufg.br/weby/up/881/o/Resolu\%C3\%A7\%C3\%A3o_Consuni.001.2021. Calend\%C3\%A1rio_2020_final.24.03.2021..pdf?1617288201>. Acesso em: 18 abr. 2021. 